Revista Brasileira de Agricultura Irrigada v.9, no .1, p.14 - 23, 2015

ISSN 1982-7679 (On-line)

Fortaleza, CE, INOVAGRI - http://www.inovagri.org.br

DOI: $10.7127 /$ rbai.v9n100272

Protocolo 272.14 - 05/11/2014 Aprovado em 24/12/2014

\title{
SUSCEPTIBILIDADE AO ENTUPIMENTO DE DIFERENTES TUBOS GOTEJADORES CONVENCIONAIS SUBMETIDOS A APLICAÇÃO DE SULFATO FERROSO VIA ÁGUA DE IRRIGAÇÃO
}

Lilian Cristina Castro de Carvalho ${ }^{1,}$ Rubens Duarte Coelho², Marconi Batista Teixeira ${ }^{3}$,

Frederico Antonio Loureiro Soares ${ }^{4}$, Fernando Nobre Cunha ${ }^{5}$, Nelmício Furtado da Silva ${ }^{6}$

\section{RESUMO}

Objetivou-se com este experimento avaliar a susceptibilidade de diferentes tubos gotejadores convencionais ao entupimento por ferro solúvel na água de irrigação (sulfato de ferroso) via duas qualidades de água (com e sem carga orgânica), sólidos suspensos (solo) e solução concentrada, com os emissores dos tubos gotejadores posicionados para baixo. O experimento foi realizado durante o período de 12 meses, com 7 modelos de tubos gotejadores. Os testes foram realizados em uma bancada de ensaios em estrutura metálica localizada no Laboratório de Irrigação, da Escola Superior de Agricultura "Luiz de Queiroz" - ESALQ/USP. A aplicação de partículas de solo e elevado teor de ferro, sem passar por filtragem intensificaram o processo de entupimento. Os modelos $\mathrm{B}, \mathrm{F}$ e $\mathrm{G}$ apresentaram entupimento total a partir das 1296, 792 e 1152 horas de funcionamento.

Palavras-chave: qualidade da água, vazão, carga orgânica, sólidos suspensos

\section{SUSCEPTIBILITY TO CLOGGING OF DIFFERENT CONVENTIONAL DRIP IRRIGATION SYSTEM SUBMITTED THE APPLICATION OF FERROUS SULFATE THROUGH IRRIGATION WATER}

\footnotetext{
ABSTRACT

The objective of this experiment was to evaluate the susceptibility of different conventional drip irrigation system to clogging by iron soluble in irrigation water (ferrous sulfate) via two

1 Professora do Instituto Federal do Ceará, IFCE. Av. Dr. Guarani, 317, Derby Clube, 62042-030, Sobral, CE. email: liliancarv@ifce.edu.br.

2 Eng. Agrônomo, Prof. Livre Docente, DER - Departamento de Engenharia Rural, Escola Superior de Agricultura Luiz de Queiroz, Universidade de São Paulo, Piracicaba, SP, C.P.: 9, CEP: 13418-900, fone (0xx19) 3447-8551, email: rdcoelho@esalq.usp.br

3 Eng. Agrônomo, Prof. Dr. Instituto Federal Goiano - IFGoiano - Rio Verde, GO, email: marconibt@gmail.com

4 Eng. Agrônomo, Prof. Dr. Instituto Federal Goiano - IFGoiano - Rio Verde, GO, email: fredalsoares@gmail.com

${ }^{5}$ Eng. Agrônomo, Doutorando em Ciências Agrárias - Agronomia, IFGoiano - Campus Rio Verde, Rio Verde, GO, email: fernandonobrecunha@hotmail.com

${ }^{6}$ Eng. Agrônomo, Doutorando em Ciências Agrárias - Agronomia, IFGoiano - Campus Rio Verde, Rio Verde, GO, email: nelmiciofurtado@gmail.com
} 
water qualities (with and without organic load), suspended solids (soil) and concentrated solution, with the imitter of the dripline positioned down. The experiment was conducted during the period of 12 months, with 7 models dripline. The tests were performed on a test bench in metal structure located in the Irrigation Laboratory of the College of Agriculture "Luiz de Queiroz" - ESALQ/USP. The application of soil particles and high iron content without going through filtering intensified clogging process. The models B, F and G showed complete clogging from the 1296, 792 and 1152 hours of operation.

Keywords: water quality, flow, organic matter, suspended solids.

\section{INTRODUÇÃO}

A qualidade da água é um fator essencial, pois problemas com entupimento diminuem a eficiência do sistema. Desta forma análises das fontes de água são extremamente necessárias, exigindo-se água de boa qualidade, livre de sólidos suspensos e dissolvidos, como o ferro, que pode precipitar e causar problemas no sistema, sendo bastante aconselhável sua análise antes da instalação do sistema de gotejamento (BRAUER, 2010).

Os agentes causadores de entupimento nas tubulações e emissores dos sistemas de irrigação podem ser de natureza física química ou biológica. Os agentes físicos causadores de entupimento são partículas inorgânicas em suspensão (areia, silte e argila) e partículas orgânicas (fragmentos vegetais e animais, bactérias, algas, larvas, etc.).

O entupimento pode ser parcial, reduzindo a uniformidade de aplicação, ou total, interrompendo por completo o funcionamento do sistema, causando sérios problemas às culturas, devido à deficiência hídrica (CARARO et al., 2006).

Nos solos o ferro pode ser encontrado na forma de hematita $\left(\mathrm{Fe}_{2} \mathrm{O}_{3}\right)$, sesquióxido de ferro $\left(\mathrm{Fe}_{2} \mathrm{O}_{3} \cdot 3 \mathrm{H}_{2} \mathrm{O}\right)$ ou siderita $\left(\mathrm{FeCO}_{3}\right)$. Segundo Vanzela (2004), o processo de erosão dos solos formados a base de sesquióxidos de ferro faz com que haja um aumento na concentração de ferro tanto solúvel quanto em suspensão nas águas.
As obstruções por microrganismos (fitoplâncton - algas e zooplâncton - bactérias) que se desenvolvem alimentando-se de resíduos orgânicos ou inorgânicos presentes nas aguas são causadas pelos filamentos dos microrganismos que crescem formando uma matriz gelatinosa e pegajosa que se adere nas partes internas da tubulação e dos emissores. Já as obstruções oriundas de material em suspensão são as de mais fácil solução, haja vista que um eficiente sistema de filtragem, pode reduzir significativamente o problema (SOUZA, 2006)

Segundo Coelho et al. (2014) a utilização de água com elevado teor de ferro em sistemas de irrigação por gotejamento têm proporcionado sérios problemas de obstrução dos emissores. Assim, torna-se imprescindível o desenvolvimento de pesquisas que possam trazer soluções práticas para serem implantadas em campo.

Objetivo com este experimento foi avaliar a susceptibilidade de diferentes tubos gotejadores convencionais ao entupimento por ferro solúvel na água de irrigação (sulfato ferroso) via duas qualidades de água (com e sem carga orgânica), sólidos suspensos (solo) e solução concentrada, com os emissores dos tubos gotejadores posicionados para baixo.

\section{MATERIAL E MÉTODOS}

A presente pesquisa foi conduzida no Laboratório de Irrigação de Irrigação da Escola 
Superior de Agricultura "Luiz de Queiroz" ESALQ/USP, localizada no município de Piracicaba - SP, cujo clima é classificado como Cwa - subtropical úmido, segundo classificação climática de Köppen, temperatura média máxima superior a $22^{\circ} \mathrm{C}$ e média mínima de $18^{\circ} \mathrm{C}$, ocorrendo chuvas de verão e secas de inverno.

$\mathrm{O}$ experimento foi desenvolvido em uma bancada de ensaios, construída em estrutura metálica composta por três andares, com $11 \mathrm{~m}$ de comprimento, $4 \mathrm{~m}$ de largura e $5,8 \mathrm{~m}$ de altura, sendo cada andar da bancada independente dos demais.

Os andares foram divididos em duas partes, sendo cada uma composta por diferentes níveis para a inserção das linhas gotejadoras que foram unidas no início e no final da bancada por registros e conexões de PVC. Para a condução da água e montagem das linhas de gotejadores do ensaio utilizou-se um registro de 2" na linha principal e um registro de $3 / 4$ " na entrada de cada nível. Na entrada de cada módulo da bancada, foi instalada uma tomada de pressão, permitindo o ajuste da pressão a cada medição de vazão, e se necessário, ajustada àquela preestabelecida. Para a medição e monitoramento da pressão de serviço, foi utilizado um manômetro com faixa de leitura de $0-700 \mathrm{kPa}$, calibrado previamente por um manômetro de peso morto.

O sistema de pressurização utilizado no experimento foi composto por um conjunto de motobomba centrífuga da marca KSB, modelo KSB Hydrobloc C 750, cuja operação de funcionamento foi realizada manualmente, obedecendo rigorosamente os horários de início e fim de cada aplicação. Para evitar a entrada de partículas em suspensão no sistema, foi utilizado um filtro de disco de $120 \mathrm{mesh} / \mathrm{Amiad}$, com capacidade para $15 \mathrm{~m}^{3} \mathrm{~h}^{-1}$ de vazão, cuja lim- peza foi feita a cada 12 horas de funcionamento.

No preparo e armazenamento das soluções utilizaram-se caixas de amianto, com capacidade para $1000 \mathrm{~L}$, que foram conectadas ao conjunto motobomba por meio de uma sucção de 0,06 $\mathrm{m}$ de diâmetro, através de um adaptador de caixa d'água e o bombeamento de cada solução foi controlado por um registro de esfera de $0,06 \mathrm{~m}$, instalado na extremidade da sucção dentro da caixa, permitindo o bombeamento da solução.

O sistema de aplicação da solução foi adaptado de tal forma que permitia um processo de recirculação da mesma, onde a solução gotejada era recolhida por dois conjuntos de telhas de aço zincado (forradas com filme plástico), com uma inclinação de $3 \%$, aonde conduzia a solução para uma calha de zinco revestida com fibra de vidro, localizada no centro do modulo, que retornava a solução para a caixa da respectiva captação, através de um conjunto de tubos PVC de $100 \mathrm{~mm}$.

Ao final da aplicação, os registros eram fechados com o sistema ainda em funcionamento, para evitar o retorno da solução ao reservatório, garantindo assim que a solução permanecesse em contato com toda a superfície interna da linha gotejadora. Para evitar uma perda acentuada da solução ao longo da semana, foram colocadas cortinas de plásticos nas laterais da bancada de ensaio. Após cada ensaio de vazão, o sistema permanecia desligado por um dia, com o objetivo de se fazer limpeza, preparo da nova solução, reparos e manutenção do sistema.

O tratamento realizado na Fase 1 do experimento pode ser resumido da seguinte forma: linhas gotejadoras do setor $1-6 \mathrm{mgL}^{-1}$ de $\mathrm{Fe}^{+2}$ (sulfato ferroso) + água LAB (Tabela 1). 
Tabela 1. Análise da água do Laboratório de Irrigação da ESALQ/USP, que foi utilizada na preparação da solução aplicada na Fase 1.

\begin{tabular}{|c|c|c|}
\hline Parâmetro & Unidade & Resultado \\
\hline $\begin{array}{c}\text { Alcalinidade } \\
\left(2 \mathrm{CO}_{3}{ }^{2-}+\mathrm{HC}_{3}^{-}\right)\end{array}$ & $\operatorname{mg~L} L^{-1}$ & 35,8 \\
\hline Cloreto $\left(\mathrm{Cl}^{-}\right)$ & $\mathrm{mg} \mathrm{L}^{-1}$ & 33,9 \\
\hline Nitrato $\left(\mathrm{N}-\mathrm{NO}_{3}\right)$ & $\mathrm{mg} \mathrm{L}^{-1}$ & 20,5 \\
\hline Sulfato $\left(\mathrm{SO}_{4}^{2-}\right)$ & $\mathrm{mg} \mathrm{L}^{-1}$ & 149,3 \\
\hline Fósforo (P) & $\mathrm{mg} \mathrm{L}^{-1}$ & 0,05 \\
\hline $\begin{array}{l}\text { Nitrogênio Amoniacal } \\
\qquad\left(\mathrm{N}-\mathrm{NH}_{3}\right)\end{array}$ & $\operatorname{mg~L}^{-1}$ & 0,11 \\
\hline Sódio $\left(\mathrm{Na}^{+}\right)$ & $\operatorname{mg~L}^{-1}$ & 44,0 \\
\hline Potássio $\left(\mathrm{K}^{+}\right)$ & $\mathrm{mg} \mathrm{L}^{-1}$ & 7,1 \\
\hline Cálcio $\left(\mathrm{Ca}^{2+}\right)$ & $\mathrm{mg} \mathrm{L}^{-1}$ & 33,2 \\
\hline Magnésio $\left(\mathrm{Mg}^{2+}\right)$ & $\mathrm{mg} \mathrm{L}^{-1}$ & 9,2 \\
\hline Ferro $\left(\mathrm{Fe}^{2+}\right)$ & $\mathrm{mg} \mathrm{L}^{-1}$ & 0 \\
\hline Cobre $(\mathrm{Cu})$ & $\mathrm{mg} \mathrm{L}^{-1}$ & 0,01 \\
\hline Manganês (Mn) & $\mathrm{mg} \mathrm{L}^{-1}$ & 0,09 \\
\hline Zinco (Zn) & $\mathrm{mg} \mathrm{L}^{1}$ & 1,14 \\
\hline Boro (B) & $\mathrm{mg} \mathrm{L}^{-1}$ & 0 \\
\hline Alumínio (Al) & $\mathrm{mg} \mathrm{L}^{-1}$ & 0 \\
\hline Cor aparente & $\mathrm{PtCo}$ & 4 \\
\hline Turbidez & FTU & 4 \\
\hline Sedimentos em suspensão & $\mathrm{mg} \mathrm{L}^{-1}$ & 6,0 \\
\hline $\begin{array}{l}\text { Condutividade elétrica } \\
\text { (CE) }\end{array}$ & $\mathrm{mS} \mathrm{cm} \mathrm{cm}^{-1}$ & 0,37 \\
\hline $\mathrm{pH}$ & ---- & 7,5 \\
\hline Gás carbônico $\left(\mathrm{CO}_{2}\right)$ & $m g L^{-1}$ & 1,9 \\
\hline Acidez $\left(\mathrm{CaCO}_{3}\right)$ & $\mathrm{mg} \mathrm{L}^{-1}$ & 6,5 \\
\hline Dureza total $\left(\mathrm{CaCO}_{3}\right)^{*}$ & $\mathrm{mg} \mathrm{L}^{-1}$ & 120,6 \\
\hline
\end{tabular}

"Dureza total calculada com base no equivalente de carbonato de cálcio $\left(\mathrm{CaCO}_{3}\right)$.

Na Fase 2, os tratamentos permaneciam 24 horas em aplicação continua e 72 horas em repouso e avaliou-se a susceptibilidade dos tubos gotejadores ao entupimento quando submetidos à aplicação de carga orgânica (fitoplâncton / algas), mais sólidos em suspensão, na concentração de $409 \mathrm{mg} \mathrm{L}^{-1}$ (Tabela 2) e 18,8 $\mathrm{mg} \mathrm{L}^{-1}$ de ferro solúvel $\left(\mathrm{Fe}^{+2}\right)$, onde se utilizou como fonte de ferro uma associação de sulfato ferroso $\left(6 \mathrm{mg} \mathrm{L}^{-1}\right)$, de óxido de ferro $\left(6 \mathrm{mg} \mathrm{L}^{-1}\right)$ e de hidróxido de ferro $\left(6,8 \mathrm{mg} \mathrm{L}^{-1}\right)$; associados apenas a água do lago e teve duração de 1068 horas de aplicação. Nesta fase (FASE 2), as linhas gotejadoras dos setores 1 tiveram seus orifícios posicionados para baixo.

Tabela 2. Características físicas do solo da área experimental

\begin{tabular}{|c|c|c|c|c|c|}
\hline \multicolumn{3}{|c|}{ Granulometria (\%) } & \multirow{3}{*}{$\begin{array}{c}\text { Classe } \\
\text { Textural } \\
\text { argilosa }\end{array}$} & \multicolumn{2}{|c|}{ Densidade $\left(\mathrm{g} \mathrm{cm}^{-3}\right)$} \\
\hline argila & silte & areia & & global & partículas \\
\hline 48,16 & 17,72 & 34,12 & & 1,45 & 2,79 \\
\hline
\end{tabular}

Ainda na Fase 2, foi aplicado uma solução concentrada com uma relação de 1:30 (30 vezes a solução do reservatório), diretamente nos tubos gotejadores, sem passar por filtragem, este procedimento fora adotado, com o objetivo de simular a quantidade de material sedimentado numa linha de tubos gotejadores instalada em campo, que em grande parte chega a medir até 300 metros, então como o comprimento da linha na bancada de ensaios era de 10 metros adotou-se a relação de 1:30. Esta solução concentrada era composta pelos mesmos elementos da solução utilizada na irrigação, sendo aplicada por aproximadamente 5 minutos, após o período de irrigação de 24 horas, e ficando em repouso dentro dos tubos gotejadores por 72 horas, até o reinício do processo de irrigação, simulando, assim, condições extremas de qualidade de água.

Os tratamentos realizados na Fase 2 pode ser resumido da seguinte forma: linhas gotejadoras do setor $1-18,8 \mathrm{mg} \mathrm{L}^{-1}$ de $\mathrm{Fe}^{+2}$ (sulfato ferroso, óxido de ferro e hidróxido de ferro) + sólidos suspensos (solo - $409 \mathrm{mg} \mathrm{L}^{-1}$ ) + carga orgânica (Tabela 3) + solução 
concentrada (30 vezes a solução do reservatório), por um período de 5 minutos, com os emissores posicionados para baixo.

Tabela 3. Análise da água do lago utilizada na preparação da solução aplicada na Fase 2

\begin{tabular}{|c|c|c|}
\hline Parâmetro & Unidade & Resultado \\
\hline $\begin{array}{c}\text { Alcalinidade } \\
\left(2 \mathrm{CO}_{3}{ }^{2-}+\mathrm{HCO}_{3}{ }^{-}\right)\end{array}$ & $m g L^{-1}$ & 88,4 \\
\hline Cloreto $\left(\mathrm{Cl}^{-}\right)$ & $\mathrm{mg} \mathrm{L}^{-1}$ & 19,0 \\
\hline Nitrato $\left(\mathrm{N}-\mathrm{NO}_{3}\right)$ & $\mathrm{mg} \mathrm{L}^{-1}$ & 0,6 \\
\hline Sulfato $\left(\mathrm{SO}_{4}{ }^{2-}\right)$ & $\mathrm{mg} \mathrm{L}^{-1}$ & 12,1 \\
\hline Fósforo (P) & $\mathrm{mg} \mathrm{L}^{-1}$ & 0,14 \\
\hline $\begin{array}{l}\text { Nitrogênio Amoniacal } \\
\qquad\left(\mathrm{N}-\mathrm{NH}_{3}\right)\end{array}$ & $\mathrm{mg} \mathrm{L}^{-1}$ & 1,1 \\
\hline Sódio $\left(\mathrm{Na}^{+}\right)$ & $\mathrm{mg} \mathrm{L}^{-1}$ & 24,0 \\
\hline Potássio $\left(\mathrm{K}^{+}\right)$ & $\mathrm{mg} \mathrm{L}^{-1}$ & 6,3 \\
\hline Cálcio $\left(\mathrm{Ca}^{2+}\right)$ & $\mathrm{mg} \mathrm{L}^{-1}$ & 17,5 \\
\hline Magnésio $\left(\mathrm{Mg}^{2+}\right)$ & $\mathrm{mg} \mathrm{L}^{-1}$ & 3,7 \\
\hline Ferro $(\mathrm{Fe})$ & $\mathrm{mg} \mathrm{L}^{-1}$ & 0,75 \\
\hline Cobre $(\mathrm{Cu})$ & $\mathrm{mg} \mathrm{L}^{-1}$ & 0 \\
\hline Manganês (Mn) & $\mathrm{mg} \mathrm{L}^{-1}$ & 0,04 \\
\hline Zinco (Zn) & $\mathrm{mg} \mathrm{L}^{-1}$ & 0,17 \\
\hline Cor Aparente & $\mathrm{mg} \mathrm{L}^{-1}$ & 230 \\
\hline Turbidez & $\mathrm{mg} \mathrm{L}^{-1}$ & 48 \\
\hline $\begin{array}{l}\text { Sedimentos em } \\
\text { suspensão }\end{array}$ & $\mathrm{PtCo}$ & 25 \\
\hline $\begin{array}{l}\text { Condutividade elétrica } \\
\text { (CE) }\end{array}$ & FTU & 0,18 \\
\hline $\mathrm{pH}$ & $\mathrm{mg} \mathrm{L}^{-1}$ & 7,4 \\
\hline Gás Carbônico $\left(\mathrm{CO}_{2}\right)$ & $\mathrm{mS} \mathrm{cm}{ }^{-1}$ & 5,3 \\
\hline Acidez $\left(\mathrm{CaCO}_{3}\right)$ & --- & 33 \\
\hline Dureza Total* $\left(\mathrm{CaCO}_{3}\right)$ & $\mathrm{mg} \mathrm{L}^{-1}$ & 58,7 \\
\hline $\begin{array}{l}\text { Coliformes } \\
\text { totais } / 100 \mathrm{~mL}\end{array}$ & $\mathrm{mg} \mathrm{L}^{-1}$ & $1,3 \times 10^{3}$ \\
\hline $\begin{array}{c}\text { Coliformes } \\
\text { termotolerantes } / 100 \mathrm{~mL}\end{array}$ & $\mathrm{mg} \mathrm{L}^{-1}$ & $9,2 \times 10^{2}$ \\
\hline $\begin{array}{l}\text { *Dureza Total calculad } \\
\text { Carbonato de cálcio }(\mathrm{C} \\
2,497[\mathrm{Ca}, \mathrm{mg} \mathrm{L}-1]+4,\end{array}$ & $\begin{array}{l}\text { 3) } \operatorname{seg} \\
{[\mathrm{Mg}, \mathrm{n}}\end{array}$ & $\begin{array}{l}\text { valente } \\
\text { on }(199\end{array}$ \\
\hline
\end{tabular}

Durante o ensaio foram mantidos os espaçamentos originais das linhas gotejadoras, com o objetivo de evitar diferenças relacionadas à influência de emendas e da proximidade dos emissores, reduzindo os erros e mantendo, assim, as mesmas condições das linhas utilizadas pelos irrigantes. Desta forma o número de gotejadores presentes na linha dependeu do espaçamento fornecido pelo fabricante, porém foi assegurado um número mínimo de 10 emissores para cada modelo analisado.

A Tabela 4 apresenta os emissores com suas características técnicas e a vazão mais comercializada pelos irrigantes, ensaiados nas Fases 1 e 2. Em função dos emissores ensaiados serem produtos comerciais e os ensaios a que foram submetidos não serem normatizados, os dados e análises realizados em todos os ensaios foram codificados para evitar qualquer tipo de especulação comercial dos resultados apresentados. A codificação utilizada na representação dos modelos de emissores, não tem nenhuma relação com os nomes comerciais dos produtos e nem tampouco com a sequência de emissores apresentados na Tabela 4.

Tabela 4. Características técnicas: fluxo, vazão, diâmetro nominal ( $\varnothing \mathrm{N})$ e espaçamento entre gotejadores dos modelos convencionais utilizados no experimento das Fases 1 e 2.

\begin{tabular}{|c|c|c|c|c|c|}
\hline \multirow[t]{4}{*}{ 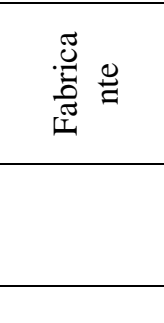 } & $\frac{\frac{0}{0}}{\frac{0}{2}}$ & 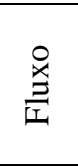 & $\begin{array}{l}\text { Vazão } \\
\left(\mathrm{L} \mathrm{h}^{-1}\right)\end{array}$ & $\begin{array}{l}\varnothing \mathrm{N} \\
(\mathrm{m})\end{array}$ & $\begin{array}{l}\text { Espaçamento } \\
\text { entre } \\
\text { gotejadores } \\
(\mathrm{m})\end{array}$ \\
\hline & Tiran & $\mathrm{NC}$ & 2,05 & 16 & 0,75 \\
\hline & $\begin{array}{c}\text { Super } \\
\text { Typhoon }\end{array}$ & $\mathrm{NC}$ & 2,0 & 16 & 0,60 \\
\hline & Hydrogol & $\mathrm{NC}$ & 3,0 & 17 & 0,40 \\
\hline Naandan & Naan Paz & $\mathrm{NC}$ & 2,0 & 16 & 0,75 \\
\hline Azud & Azudline & $\mathrm{NC}$ & 1,4 & 17 & 0,50 \\
\hline Petroísa & $\begin{array}{c}\text { Petroísa } \\
\text { G1 }\end{array}$ & $\mathrm{NC}$ & 1,5 & 16 & 0,50 \\
\hline Metzerplas & Vered & $\mathrm{NC}$ & 2,1 & 17 & 0,50 \\
\hline \multicolumn{6}{|c|}{$\begin{array}{l}\text { *Gotejadores convencio } \\
\text { Catálogo dos fabricantes }\end{array}$} \\
\hline \multicolumn{6}{|c|}{$\begin{array}{l}\text { procederam-se os cálculos da vazão, vazão } \\
\text { relativa e do coeficiente de variação de vazão, } \\
\text { através das Equações de } 1 \text { a } 3 \text {. }\end{array}$} \\
\hline
\end{tabular}




$$
q=\frac{P}{1000 t d} 60
$$

em que:

$\mathrm{q}$ - vazão do gotejador, $\mathrm{L} \mathrm{h}^{-1}$;

$\mathrm{P}$ - peso da água coletada, $\mathrm{g}$;

$\mathrm{t}$ - tempo de coleta, min e

$d$ - densidade da água utilizada no ensaio, $\mathrm{g} \mathrm{L}^{-1}$

(o valor médio utilizado para conversão da água para $25^{\circ} \mathrm{C}$, foi de 0,997$)$.

$$
Q_{r}=\frac{Q_{x, y}}{Q_{i}}
$$

em que:

$\mathrm{Q}_{\mathrm{r}}$ - vazão relativa, \%;

$\mathrm{Q}_{\mathrm{x}, \mathrm{y}}$ - vazão de um emissor $\mathrm{x}$ num dia $\mathrm{y}$ de irrigação, $\mathrm{L} \mathrm{h}^{-1} \mathrm{e}$

$\mathrm{Q}_{\mathrm{i}}$ - vazão desse emissor no primeiro dia de irrigação, $\mathrm{L} \mathrm{h}^{-1}$.

$$
C V=\frac{\sigma}{q_{m}} 100
$$

em que:

$C V$ - coeficiente de variação $(\%)$;

$\sigma-$ desvio padrão da vazão média do emissor $\left(\mathrm{L} \mathrm{h}^{-1}\right) \mathrm{e}$

$q_{m}$ - vazão média a determinada pressão e temperatura padrão $\left(\mathrm{L} \mathrm{h}^{-1}\right)$.

Os dados obtidos foram submetidos à análise de variância pelo teste $\mathrm{F}$ ao nível de $1 \mathrm{e}$ $5 \%$ de probabilidade, e em casos de significância, as médias foram comparadas entre si pelo teste Tukey à 5\% de probabilidade.

\section{RESULTADOS E DISCUSSÃO}

A vazão média dos emissores foi utilizada para se quantificar as principais alterações quanto ao funcionamento adequado dos emissores, tipo gotejadores, avaliados, de acordo com os trabalhos pertinentes (GILBERT et al.,1979; NAKAYAMA et al.,1977; RAVINA et al., 1992).

$\mathrm{Na}$ Figuras 1 encontra-se a vazão média para os diferentes modelos de gotejadores convencionais nas Fases 1 e 2 do experimento, avaliados no período do ensaio.
Conforme se observa na Figura 1 os emissores avaliados apresentaram comportamentos diferenciados quanto à redução de vazão, em função dos tratamentos impostos, decorrente da arquitetura de cada gotejador, concordando com resultados observados por Costa (2000), Teixeira (2006) e Ribeiro (2008), onde as características construtivas são diferentes em cada modelo de gotejadores fazendo com que a intensidade de entupimento mostre-se de forma heterogenia. Observa-se também, nesta Figura, que não houve grande variação nas vazões dos emissores estudados, durante a primeira Fase do experimento.

Os modelos A, D, E e F, na Fase 1 demonstraram aumentos de vazão de 5,$0 ; 0,5 ; 2,9$ e $4,7 \%$, nas $216,288,216$ e 288 horas de funcionamento respectivamente, enquanto que para os modelos B, C e G foram observadas apenas reduções de vazão, as quais foram mais expressivas nas 288,72 e 72 horas de funcionamento, sendo estas da ordem de 4,1; 10 e $6,8 \%$, respectivamente.

$\mathrm{Na}$ Fase 2 do experimento (após 360 h) observou-se grandes reduções na vazão dos gotejadores. Os modelos $\mathrm{F}$ e $\mathrm{G}$ foram os mais afetados durante o período de ensaio, chegando a uma obstrução total de seus emissores na Fase 2, a partir de 792 e 1152 horas de funcionamento, respectivamente. Os modelos $\mathrm{B}$ e $\mathrm{E}$ também apresentaram redução total nas vazões de seus emissores na Fase 2, a partir da hora 1296.

Costa (2000), trabalhando com gotejadores de diferentes fabricantes, abastecidos com água contendo teor de ferro de $3,0 \mathrm{mg} \mathrm{L}^{-1}$, observou que o modelo Naan, após 375 horas de funcionamento, sofreu uma redução de vazão de $46 \%$ e o que o modelo Netafim apresentou uma redução média de vazão de $34 \%$ logo após o início dos testes, entre 3 e 32 horas de funcionamento. 


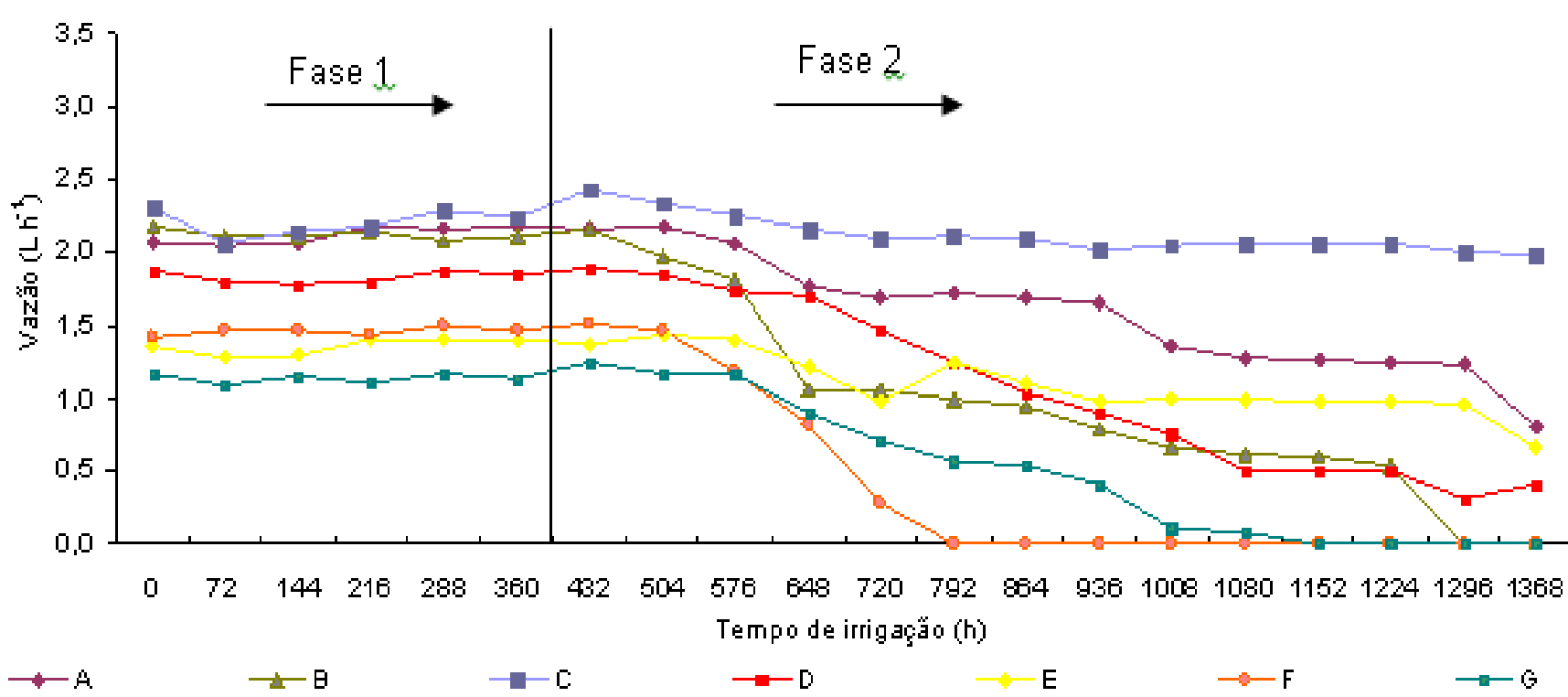

Figura 1. Vazão média dos gotejadores convencionais na Fase 1 - água LAB/6 $\mathrm{mg} \mathrm{L}^{-1} \mathrm{de} \mathrm{Fe}^{2+}$ (sulfato ferroso)

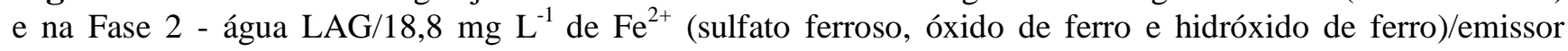
$\uparrow /$ sólidos suspensos/solução concentrada.

O posicionamento para baixo dos orifícios dos emissores propiciou uma maior susceptibilidade ao entupimento, provavelmente devido a uma maior deposição dos tratamentos no fundo do tubo gotejador (decantação), facilitando assim a obstrução dos emissores.

Na Tabela 5 encontra-se o teste de Tukey, a 5\% de probabilidade, para a comparação das médias de cada emissor ao longo do tempo, para a Fase 1 e 2 respectivamente. Conforme observado na Tabela não houve diferença significativa pelo teste de Tukey ao nível de 5\% de probabilidade, para o modelo $\mathrm{C}$.

De acordo com o Teste de Tukey, ao nível de 5\% de probabilidade, com relação à comparação de médias para cada emissor ao longo do tempo, os modelos A e E, diferiram estatisticamente entre as primeiras leituras e as últimas leituras, sendo que as leituras intermediárias não diferiram de nenhuma. Para Vieira et al. (2004) vários fatores podem comprometer a uniformidade de distribuição de água em sis- temas localizados destacando-se, entre eles, o entupimento ou o desequilíbrio de vazão dos emissores.

O modelo, $\mathrm{F}$ apresenta comportamento semelhante, pois diferiram estatisticamente de forma abrupta em três pontos: começo, meio e fim das leituras, sendo iguais de 0 a $504 \mathrm{~h}$, de 576 a 648 h e de 720 a 1368 h para o primeiro caso e de 0 a 1152 h, na leitura de 1224 h e de 1296 a $1368 \mathrm{~h}$ para o segundo caso.

$\mathrm{O}$ modelo $\mathrm{G}$ diferiu em 5 médias, apresenta grande amplitude com vazão igual a zero nas leituras finais e tem desempenho distinto em 4 ocasiões: de 0 a 648 h com alta vazão, de 720 a 936 h com vazão média, de 1008 a 1080 h com baixa vazão e de 1152 a 1368 h com obstrução total dos gotejadores.

Os modelos B e D apresentam grande amplitude entre as médias, sendo que o modelo B a partir das $1296 \mathrm{~h}$ tem vazão zero, diferem muito entre as leituras e tem um desempenho decrescente ao longo do tempo. 
Tabela 5. Vazão média $\left(\mathrm{L} \mathrm{h}^{-1}\right)$, dos modelos A, B, C, D, E, F e G convencionais na Fase 1 e 2.

\begin{tabular}{|c|c|c|c|c|c|c|c|c|}
\hline \multicolumn{9}{|c|}{ Modelos } \\
\hline Aplicação & (h) & A & B & $\mathrm{C}$ & $\mathrm{D}$ & $\mathrm{E}$ & $\mathrm{F}$ & $\mathrm{G}$ \\
\hline \multirow{6}{*}{ FASE 1} & 0 & $2,07 \mathrm{~b}$ & $2,17 f$ & $2,30 \mathrm{a}$ & $1,87 \mathrm{de}$ & $1,36 \mathrm{~b}$ & $1,42 \mathrm{c}$ & $1,17 \mathrm{de}$ \\
\hline & 72 & $2,05 \mathrm{~b}$ & $2,12 \mathrm{ef}$ & $2,07 \mathrm{a}$ & $1,80 \mathrm{de}$ & $1,27 \mathrm{ab}$ & $1,46 \mathrm{c}$ & $1,09 \mathrm{de}$ \\
\hline & 144 & $2,06 \mathrm{~b}$ & $2,12 \mathrm{ef}$ & $2,15 \mathrm{a}$ & $1,78 \mathrm{de}$ & $1,29 \mathrm{ab}$ & $1,46 \mathrm{c}$ & $1,14 \mathrm{de}$ \\
\hline & 216 & $2,18 \mathrm{~b}$ & $2,15 \mathrm{ef}$ & $2,17 \mathrm{a}$ & $1,80 \mathrm{de}$ & $1,40 \mathrm{~b}$ & $1,43 \mathrm{c}$ & $1,11 \mathrm{de}$ \\
\hline & 288 & $2,16 \mathrm{~b}$ & $2,08 \mathrm{def}$ & $2,29 a$ & $1,88 \mathrm{e}$ & $1,40 \mathrm{~b}$ & $1,49 \mathrm{c}$ & $1,16 \mathrm{de}$ \\
\hline & 360 & $2,17 \mathrm{~b}$ & $2,11 \mathrm{def}$ & $2,24 \mathrm{a}$ & $1,84 \mathrm{de}$ & $1,40 \mathrm{~b}$ & $1,46 \mathrm{c}$ & $1,13 \mathrm{de}$ \\
\hline \multirow{14}{*}{ FASE 2} & 432 & $2,16 b$ & $2,16 \mathrm{ef}$ & $2,43 a$ & $1,89 \mathrm{e}$ & $1,37 b$ & $1,51 \mathrm{c}$ & $1,24 \mathrm{e}$ \\
\hline & 504 & $2,18 b$ & $1,97 \mathrm{cdef}$ & $2,33 a$ & $1,84 \mathrm{de}$ & $1,43 b$ & $1,46 \mathrm{c}$ & $1,17 \mathrm{de}$ \\
\hline & 576 & $2,07 \mathrm{~b}$ & $1,81 \mathrm{bcdef}$ & $2,26 a$ & $1,74 \mathrm{de}$ & $1,40 \mathrm{~b}$ & $1,18 b c$ & $1,17 \mathrm{de}$ \\
\hline & 648 & 1,76ab & $1,05 \mathrm{abcde}$ & $2,16 \mathrm{a}$ & 1,71cde & $1,21 \mathrm{ab}$ & $0,82 b$ & $0,91 \mathrm{cde}$ \\
\hline & 720 & 1,69ab & $1,05 \mathrm{abcde}$ & $2,09 \mathrm{a}$ & $1,47 \mathrm{bcde}$ & $0,97 \mathrm{ab}$ & $0,28 \mathrm{a}$ & $0,70 \mathrm{~cd}$ \\
\hline & 792 & $1,72 \mathrm{ab}$ & 1,00abcd & $2,12 \mathrm{a}$ & 1,25 abcde & $1,24 \mathrm{ab}$ & $0,00 \mathrm{a}$ & $0,56 b c$ \\
\hline & 864 & 1,69ab & $0,95 \mathrm{abc}$ & $2,10 \mathrm{a}$ & 1,04abcde & $1,10 \mathrm{ab}$ & $0,00 \mathrm{a}$ & $0,53 b c$ \\
\hline & 936 & 1,66ab & $0,79 \mathrm{ab}$ & $2,02 \mathrm{a}$ & 0,90abcd & $0,98 \mathrm{ab}$ & $0,00 \mathrm{a}$ & $0,40 \mathrm{abc}$ \\
\hline & 1008 & $1,36 a b$ & $0,66 \mathrm{a}$ & $2,05 \mathrm{a}$ & $0,75 a b c$ & $1,00 \mathrm{ab}$ & $0,00 \mathrm{a}$ & $0,11 \mathrm{ab}$ \\
\hline & 1080 & $1,27 \mathrm{ab}$ & $0,61 \mathrm{a}$ & $2,06 \mathrm{a}$ & $0,51 \mathrm{ab}$ & $0,99 \mathrm{ab}$ & $0,00 \mathrm{a}$ & $0,08 \mathrm{ab}$ \\
\hline & 1152 & 1,26ab & $0,60 \mathrm{a}$ & $2,06 \mathrm{a}$ & $0,51 \mathrm{ab}$ & $0,98 \mathrm{ab}$ & $0,00 \mathrm{a}$ & $0,00 \mathrm{a}$ \\
\hline & 1224 & $1,25 \mathrm{ab}$ & $0,53 \mathrm{a}$ & $2,07 \mathrm{a}$ & $0,50 \mathrm{ab}$ & $0,97 \mathrm{ab}$ & $0,00 \mathrm{a}$ & $0,00 \mathrm{a}$ \\
\hline & 1296 & $1,23 \mathrm{ab}$ & $0,00 \mathrm{a}$ & $2,01 \mathrm{a}$ & $0,31 \mathrm{a}$ & $0,96 a b$ & $0,00 \mathrm{a}$ & $0,00 \mathrm{a}$ \\
\hline & 1368 & $0,80 \mathrm{a}$ & $0,00 \mathrm{a}$ & $1,99 \mathrm{a}$ & $0,41 \mathrm{a}$ & $0,67 \mathrm{a}$ & $0,00 \mathrm{a}$ & $0,00 \mathrm{a}$ \\
\hline
\end{tabular}

"Média com a mesma letra minúscula na coluna ao longo do tempo não indica diferença significativa pelo teste Tukey, a $5 \%$ de probabilidade. ${ }^{1}$ Fase 1 - água LAB/6 $\mathrm{mg} \mathrm{L}^{-1} \mathrm{de} \mathrm{Fe}^{2+}$ (sulfato ferroso) e Fase 2 - água LAG/18,8 $\mathrm{mg} \mathrm{L}^{-1} \mathrm{de} \mathrm{Fe}^{2+}$ (sulfato ferroso, óxido de ferro e hidróxido de ferro)/emissor $\uparrow /$ sólidos suspensos/solução concentrada.

A Tabela 6 são apresentados os valores da vazão relativa obtidos pela coleta individual de vazão dos emissores ao longo da linha. $\mathrm{Na}$ Tabela 6 pode-se observar que em alguns modelos de emissores, pequenas oscilações na vazão relativa, que em conformidade com Tei- xeira (2006), se deve provavelmente, às obstruções aleatórias que ocorriam pelo efeito de alguma eventual sobrepressão no sistema e/ou de uma influência mais acentuada da variação de temperatura na vazão (alteração da viscosidade da água) durante o período de ensaio. 
Tabela 6. Vazão Relativa (QR) dos gotejadores convencionais avaliados, no período do ensaio, para os modelos A, B, C, D, E, F e G na Fase 1 e 2 .

\begin{tabular}{ccccccccc}
\hline & \multicolumn{7}{c}{ Modelos } \\
\cline { 3 - 9 } Aplicação (h) & A & B & C & D & E & F & G \\
\cline { 2 - 9 } & 0 & 100 & 100 & 100 & 100 & 100 & 100 & 100,00 \\
FASE 1 & 72 & 99 & 98 & 90 & 96 & 94 & 103 & 93,20 \\
& 144 & 100 & 98 & 94 & 95 & 95 & 103 & 97,67 \\
& 216 & 105 & 99 & 95 & 97 & 103 & 101 & 94,54 \\
& 288 & 105 & 96 & 100 & 101 & 103 & 105 & 99,12 \\
& 360 & 105 & 97 & 97 & 99 & 103 & 103 & 96,83 \\
\hline & 432 & 104 & 100 & 106 & 101 & 101 & 107 & 106,09 \\
& 504 & 105 & 91 & 101 & 99 & 105 & 103 & 100,18 \\
& 576 & 100 & 84 & 98 & 93 & 103 & 83 & 99,65 \\
& 648 & 85 & 49 & 94 & 92 & 89 & 58 & 77,34 \\
& 720 & 82 & 48 & 91 & 79 & 72 & 20 & 59,95 \\
FASE 2 & 792 & 83 & 46 & 92 & 67 & 91 & 0 & 48,13 \\
& 864 & 82 & 44 & 91 & 56 & 81 & 0 & 45,58 \\
& 936 & 80 & 37 & 88 & 48 & 73 & 0 & 34,40 \\
1008 & 66 & 30 & 89 & 40 & 74 & 0 & 9,63 \\
1080 & 61 & 28 & 90 & 28 & 73 & 0 & 7,22 \\
& 1152 & 61 & 28 & 90 & 27 & 72 & 0 & 0,00 \\
1224 & 61 & 24 & 90 & 27 & 72 & 0 & 0,00 \\
1296 & 59 & 0 & 88 & 17 & 70 & 0 & 0,00 \\
1368 & 39 & 0 & 87 & 22 & 50 & 0 & 0,00 \\
\hline
\end{tabular}

${ }^{1} \overline{\text { Fase } 1 \text { - água LAB/6 mg L }}{ }^{-1} \mathrm{de} \mathrm{Fe}^{2+}$ (sulfato ferroso) e Fase 2 - água LAG/18,8 $\mathrm{mg} \mathrm{L}^{-1} \mathrm{de} \mathrm{Fe}^{2+}$ (sulfato ferroso, óxido de ferro e hidróxido de ferro)/emissor $\uparrow /$ sólidos suspensos/solução concentrada.

Os modelos A, B, D, E, e G na Fase 1 mostraram um comportamento bastante estável, indicando uma redução na vazão relativa de no máximo 1, 4, 5, 6 e 6,8\%, ocorridas nas 72, 288, 144, 72 e 72 horas de funcionamento respectivamente.

Os modelos $B, F$ e $G$ na Fase 2 apresentaram vazão relativa igual a zero, nas 1296, 792 e 1152 horas de funcionamento respectivamente. A obstrução total nos modelos B e F ocorreram de forma bastante abrupta, fato este que pode ser verificado na vazão relativa que antecede o entupimento (1224 e $720 \mathrm{~h}$ ), as quais para estes modelos foram de 24 e $20 \%$ respectivamente, mesmo o modelo $\mathrm{G}$ indicando apenas uma redução de 7,2\% (entre as 1080 e 1052 h) e logo uma redução um pouco menos expressiva, este modelo já dava sinais de uma provável ocorrência de obstrução total, com reduções na vazão relativa equivalentes as observadas nos modelos B e F, as quais foram encontradas entre as 576 e $648 \mathrm{~h}$, entre 648 e $720 \mathrm{~h}$ e entre as $936 \mathrm{e} 1008 \mathrm{~h}$ de funcionamento, sendo estas reduções de 22,3, 17,4 e $24,8 \%$ respectivamente.

Considerando esta redução abrupta na vazão relativa como um indicativo da ocorrência de obstrução total verifica-se que os modelos A e E, também apresentam grande susceptibilidade ao entupimento por demonstrar diferenças na vazão relativa equivalentes aquelas ocorridas nos modelos B, F e G, sendo esta redução de $20 \%$ para ambos os modelos A e E, sucedidas entre as 1296 e 1368 horas de funcionamento respectivamente. Cunha et al. (2014) concluíram que a dispersão das partículas sólidas no diâmetro interno tem maior influência na uniformidade conforme diminui o número de pontos avaliados e aumenta a obstrução total, sendo a uniformidade bastante variável quando sob obstrução parcial.

$\mathrm{O}$ modelo $\mathrm{C}$ foi $\mathrm{o}$ que melhor se comportou, ao longo de todo o ensaio, mantendo a vazão relativa de seus emissores praticamente constante, acima de $87 \%$ tanto na Fase 1 quanto na Fase 2. O modelo A, entretanto não apresentou bons resultados, na Fase 2 onde teve uma redução de $61 \%$ de sua vazão após 1368 horas de funcionamento.

\section{CONCLUSÕES}

A aplicação de partículas de solo e elevado teor de ferro, sem passar por filtragem intensificaram o processo de entupimento.

Os modelos B, F e G apresentaram entupimento total a partir das 1296, 792 e 1152 horas de funcionamento.

O posicionamento para baixo dos orifícios dos emissores propiciou uma maior susceptibilidade ao entupimento.

$\mathrm{O}$ modelo $\mathrm{C}$ foi o que apresentou um melhor desempenho, com vazão relativa de 
87\%. Este emissor pode contribuir para projetos de irrigação mais econômicos, pois não requer sistema de filtragem sofisticado.

Os modelos de emissores A e D foram os que sofreram maiores percentuais de entupimento parcial quando sob aplicação de ferro solúvel na água de irrigação, com valores de vazão relativa inferiores a $40 \%$.

\section{REFERÊNCIAS BIBLIOGRÁFICAS}

BRAUER, R. L. Dinâmica de entupimento de gotejadores em função da aplicação de água rica em ferro. Botucatu, 2010. 61p. Dissertação (Mestrado em Agronomia/Irrigação e Drenagem) - Faculdade de Ciências Agronômicas, Universidade Estadual Paulista.

CARARO, D.C.; BOTREL, T.A.; HILLS, D.J.; LEVERENZ, H.L. Analysis of clogging in drip emitters during wastewater irrigation. Applied Engineering in Agriculture, St. Joseph, v.22, n.2, p.251-257, 2006.

COELHO, R. D.; TEIXEIRA, M. B.; RIBEIRO, P. H. P.; CARVALHO, L. C. C.; CUNHA, F. C.; SILVA, N. F. Distúrbios de vazão em gotejadores devido o uso de água com presença de ferro. Revista Brasileira de Agricultura Irrigada, v.8, $\mathrm{n}^{\circ} .3$, p. 234 - 246, 2014.

COSTA, C.C. Entupimento de gotejadores provocado pela presença de ferro na água de irrigação. 2000. 85 p. Dissertação (Mestrado em Irrigação e Drenagem) - Universidade Federal de Lavras, Lavras, 2000.

CUNHA, F. N.; SILVA, N. F.; TEIXEIRA, M. B.; RIBEIRO, P. H. P.; MOURA, L. M. F.; SANTOS, C. C. Repetibilidade de um sistema de irrigação por gotejamento sob entupimento. Revista Brasileira de Agricultura Irrigada, v.8, no. 4, p.343 - 353, 2014.

GILBERT, R.G.; NAKAYAMA, F.S.; BUCKS, D.A. Trickle irrigation: prevention of clogging. Transactions of the ASAE, St. Joseph, v. 22, n. 3, p. 133-148, 1979.

NAKAYAMA, F.S.; BUCKS, D.A.; FRENCH, O.F. Reclaiming partially clogged trickle emitters. Transactions of the ASAE, St. Joseph, v. 20, p. 278-280, 1977.

RAVINA, I.; PAZ, E.; SOFER, Z.; MARCU, A.; SCHISCHA, A.; SAGI, G. Control of emitter clogging in drip irrigation with reclaimed wastewater. Irrigation Science, New York, v. 13, p. 129-139, 1992.

RIBEIRO, T. A. P.; PATERNIANI, J. E. S.; COLETTI, C. Chemical treatment to unclogg dripper irrigation systems due to biological problems. Scientia Agrícola, Piracicaba, v.65, n.1, p.1-9, 2008.

SOUZA, J. A. A.; CORDEIRO, E. A.; COSTA, E. L. Aplicação de hipoclorito de sódio para recuperação de gotejadores entupidos em irrigação com água ferruginosa. Revista Brasileira de Engenharia Agrícola e Ambiental, Campina Grande, v. 10, n. 1, p. 59, 2006.

TEIXEIRA, M.B. Efeitos de dosagens extremas de cloro e pH na vazão de gotejadores autocompensantes (irrigação localizada). 2006. 322 p. Tese (Doutorado em Irrigação e Drenagem) - Escola Superior de Agricultura "Luiz de Queiroz", Universidade de São Paulo. Piracicaba, 2006.

VANZELA, L. S. Qualidade de água para irrigação na microbacia do Córrego Três Barras no Município de Marinópolis. 2004. 105 p. Dissertação (Mestrado em Sistema de Produção) - Faculdade de Engenharia de Ilha Solteira. Universidade Estadual paulista "Júlio de Mesquita Filho”, Ilha Solteira, 2004.

VIEIRA, G. H. S.; MANTOVANI, E. C., CORDEIRO, É. A. Estudo comparativo da avaliação de sistemas de irrigação por gotejamento utilizando os métodos propostos por Keller e por Denículi. PNP\&D-café, EMBRAPA Café. Viçosa-MG, p.15. 2004. 